\title{
Simulation of Endovascular Neurointervention Using Silicone Models: Imaging and Manipulation
}

\author{
Yasuhiro SUZUKI, Mitsuyuki FUjITSUKA, and John C. CHALOUPKA \\ Department of Radiology, Interventional Neuroradiology Service, \\ University of Iowa Hospitals and Clinics, Iowa City, Iowa, U.S.A.
}

\begin{abstract}
Silicone models of cerebral aneurysm and stroke pathology were produced by copying the images of clinical cases to evaluate the simulation of endovascular procedures. Conventional silicone models, silicone suture models made from separate aneurysm sac and parent artery sutured together, and animal vascular models made from vessels obtained from pigs were made. The models were incorporated in the training system for simulation of endovascular procedures under digital subtraction angiography control using a portable angiography system. The conventional silicone models provided very close reproduction of the aneurysm and vessels, even the fine branches. However, greater resistance or impact was felt, especially when passing the device through curved vessels. Stroke models were insufficiently dilated by percutaneous transluminal angioplasty or stenting. The silicone suture model was useful to avoid damage to models of giant aneurysm or models with small orifices during the lost wax procedure, whereas the projection of domes was somewhat inaccurate. The animal vascular model could not provide accurate aneurysm shape and vessel diameter, although the feel during manipulation was much more realistic.
\end{abstract}

Key words: training, silicone, endovascular procedure, cerebral aneurysm, stroke

\section{Introduction}

Neurointerventional therapy has rapidly developed with the introduction of the Guglielmi detachable coil $^{3,4)}$ to treat cerebral aneurysms and the stent to treat stroke pathologies. ${ }^{7,8,10)}$ Training in these techniques involves a steep learning curve, with the most improvement seen during the first 100-200 procedures. $^{7,9)}$ The rapid development and wider use of neurointervention have increased the need for realistic training programs for endovascular techniques. More surgical experience is the basis for improvement of skills, but additional training programs can also contribute to the training of interventional neuroradiologists if circumstances restrict clinical experience. In addition, the introduction of new devices or particularly difficult cases are a valid target for other methods, even for proficient neurosurgeons.

Artificial vascular models are widely employed for research, clinical training, and preclinical evaluation of devices and implants. ${ }^{1,2,9,12)}$ Models made from non-elastic substances may simulate the shape and size of intracranial aneurysms but lack the unique properties of cerebral aneurysms, such as the thin and vulnerable wall. ${ }^{2)}$ Human vascular models are usually made from silicone by the lost wax technique, which allows an exact copy of the vasculature derived from a cadaver. ${ }^{5}$ Such a model corresponds to the individual variation of vascular courses and pathologies of the donor, but does not necessarily reflect any particular variation of interest, especially in cases of unusual or difficult anatomy.

The present study attempted to produce silicone models based on the images of clinical cases, and evaluated the uses of such silicone models by angiography and the simulation of endovascular procedures.

\section{Methods}

\section{Manufacture of models}

Three types of models were prepared: convention-

Received September 21, 2004; Accepted April 12, 2005

Author's present address: Y. Suzuki, M.D., Department of Neurosurgery, Ikegami General Hospital, Tokyo, Japan. 


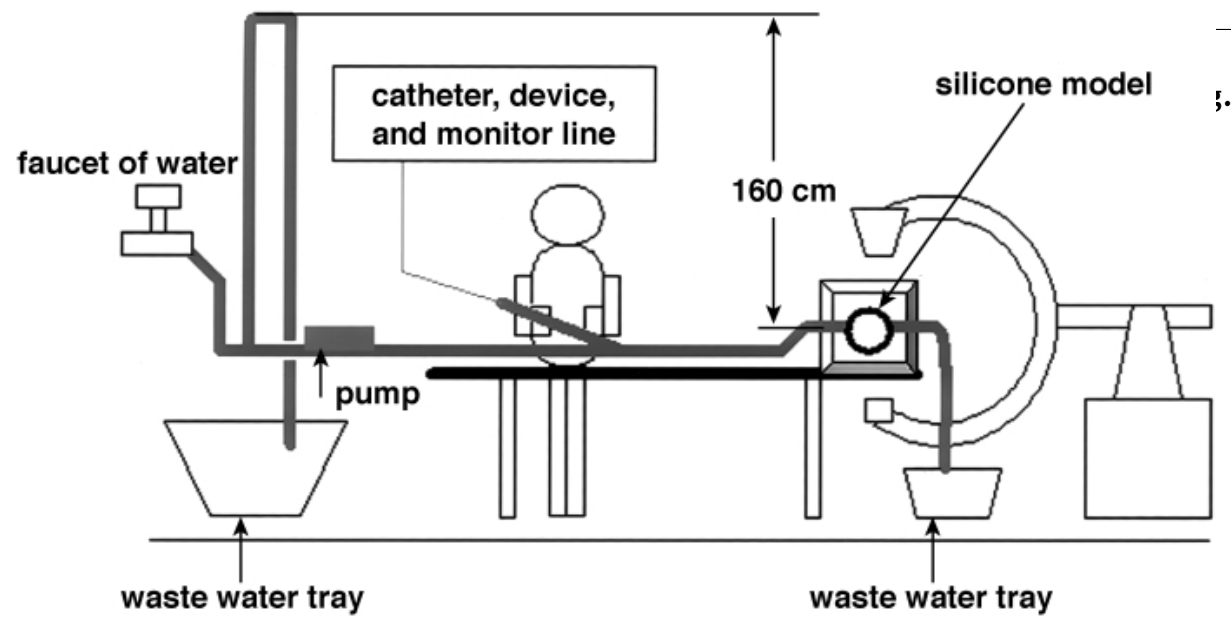

5. 1 Training arrangement. The water flows from the faucet to the discharge tray through a rotatory pump. The side tube before the rotatory pump was set to a level of $160 \mathrm{~cm}$ above the model, so that the pressure in the main tube was equivalent to about $120 \mathrm{mmHg}$. Pulsatile flow and pressure were reproduced by the rotatory pump, and the systolic, diastolic, and mean pressure, and the pulsation rate were measured proximal to the aneurysm. Another side tube was used to introduce the intraluminal catheter and microcatheter to the aneurysm.

al silicone models of cerebral aneurysms and stroke pathologies, silicone suture models with the aneurysm sac and the parent artery sutured together, and animal vascular models with vessels obtained from pigs.

Conventional silicone model: Multiple projection angiographic images (pre- and post-endovascular procedure) of clinical cases were used for the model planning. The wax models were made from wax wire of the stick (diameter $5 \mathrm{~mm}$ and $3 \mathrm{~mm}$; J.F. McCaughin Co., Rosemead, Calif., U.S.A.) and bobbin types (diameter 14, 15, and 16 gauge; Kewax $^{\mathbb{R}}$; Keystone Products Co., Philadelphia, Pa., U.S.A.). Aneurysms were formed and the diameter checked with calipers. The aneurysms and branch vessels were then heated with an iron bar for bonding and united with the parent artery.

Prosthetic silicone elastomer was prepared by mixing the three components (Silastic $^{\mathrm{R}}$, MDX4-4210, Medical Grade Elastomer $210 \mathrm{ml}$, Curing Agent $24 \mathrm{ml}$, and 200 Fluid $147 \mathrm{ml}$; Dow Corning Co., Midland, Mich., U.S.A.) in a plastic container and standing under a vacuum for about 45 minutes or until all bubbles had disappeared. The wax models were dipped into the liquid and dried out (24 hours). The ends of the parent artery and branches were then cut, the assembly dipped in tepid water, and the wax was removed by squeezing with care.

Common pathologies which are frequently treated by endovascular neurointervention procedures were selected based on actual cases as follows. The aneurysm models included the internal carotidposterior communicating artery (IC-PcomA), inter- nal carotid-ophthalmic artery (IC-OphA), cavernous portion of the internal carotid artery (cavernous ICA), anterior communicating artery (AcomA), middle cerebral artery (MCA), and top of the basilar artery (BA top). The stroke models included stenosis of the cervical ICA, embolic occlusion of the carotid bifurcation using muscle, clay, and clot as the embolic material, and stenosis of the MCA.

Silicone suture model: The silicone suture model, in which the aneurysm sac and parent artery were separately made and then sutured, was adopted for narrow neck and giant aneurysms, and aneurysms with a complicated vascular course, such as ICOphA (narrow neck) and cavernous ICA giant aneurysms. The components were sutured with 7-0 nylon under the microscope and the surface was coated with repellent.

Animal vascular model: The animal vascular model was used to model various pathologies including IC-OphA, BA top, and AcomA aneurysms. The common carotid artery and the internal jugular (diameter $3 \mathrm{~mm}$ ), external jugular $(5-7 \mathrm{~mm}$ ), or brachiocephalic vein $(10 \mathrm{~mm})$ were harvested from pigs which were euthanized immediately after the use of another experiment and dipped in saline. Foregoing research was to evaluate the effect of hypothermia for resuscitation after cardiac arrest, under general anesthesia with mechanical ventilation. There was no surgical injure except the cannulation of bilateral femoral veins and unilateral artery. A silicone model employed as a sheath was made beforehand to reproduce the curve of the vessels. The aneurysm and branches were formed by anastomosing the arteries and veins using 7-0 nylon 
under the microscope. The artery model was then placed in the silicone sheath. A hole corresponding to the size of the aneurysm neck was made in the silicone wall corresponding to the aneurysm. After 4 or 5 French sheaths were cut to adequate lengths, the tips were inserted in the ends of silicones and arteries, and then fastened tightly by thread.

\section{Training system}

The training system approximated as realistically as possible to the clinical endovascular treatment procedure (Fig. 1). Digital subtraction angiography and monitoring of the endovascular procedure used a portable angiography system with road mapping function. Water flowed from the faucet to the discharge tray through a rotatory pump. The side tube was set to a level of $160 \mathrm{~cm}$ above the model, so that the pressure in the main tube was equivalent to about $120 \mathrm{mmHg}$. Pulsatile flow and pressure were reproduced by the rotatory pump, and the systolic, diastolic, and mean pressure, and the pulsation rate were measured proximal to the aneurysm. Another side tube was used to introduce the intraluminal 7 French catheter and microcatheter to the aneurysm. The procedure was recorded by two video cameras (perspective image and direct view) and transmitted to a video-monitor, where the investigator could follow the course of the intervention.

\section{Evaluation}

Conventional silicone models were examined from the anterior-posterior and lateral directions, and the angiographical views employed during clinical treatment, and the images were compared with those of the original case. In the case of an aneurysm, the tip of the microcatheter was advanced into the dome, and the first coil was released. In the case of a stenting procedure, after deploying the stent, coil release was performed according to the clinical procedure. In the case of stroke pathology, the stent was deployed for cervical ICA stenosis after percutaneous transluminal angioplasty (PTA). In the case of embolic occlusion of the carotid bifurcation, the microcatheter was passed through the occlusion and angiography of the distal portion was performed. In the case of MCA stenosis, PTA was performed. The subjective realism of the procedure was evaluated compared with that of clinical manipulation. Silicone suture models and animal vascular models were also examined to evaluate the realism compared with clinical manipulation.

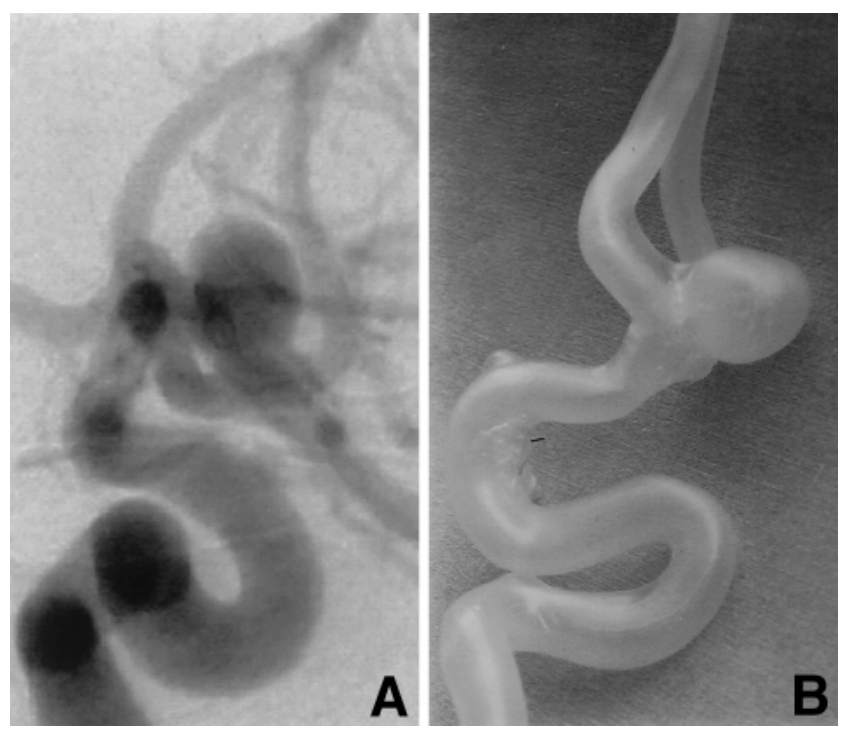

Fig. 2 Angiograms of an anterior communicating artery aneurysm (A) and the silicone model (B) showing close matching despite some insignificant differences.

\section{Results}

\section{Conventional silicone model}

A uniform thickness of wall was difficult to produce, because the silicon liquid tended to shrink at the beginning of drying. Any remaining bubbles tended to form pores which caused leakages. Inspection of the lumen of the model found that the inner surface was not as smooth as the outer surface, and web or inequalities were observed around the neck of the aneurysm or stenosis.

The aneurysm model closely reproduced the original anatomy, despite some insignificant differences (Figs. 2 and 3A-C). Even the fine branches such as the ophthalmic artery and superior cerebellar artery could be reproduced (Fig. 3B, C). However, narrow neck aneurysms and branches such as the MCA or PcomA were occasionally lost when the wax models were dipped into silicone liquid. The procedure of eliminating the wax from aneurysms with large size or narrow neck was time consuming, so the silicone wall was likely to tear.

Silicone has a higher coefficient of friction compared to actual living vessels and so the resistance to the introduction of devices is stronger, especially when passing various devices through curved vessels (Fig. 3D). Moreover, the feel of coiling was very different from practical manipulation. In some cases, the microcatheter or stent did not pass the carotid siphon or the junction between the BA and posterior cerebral artery. Resistance could be 


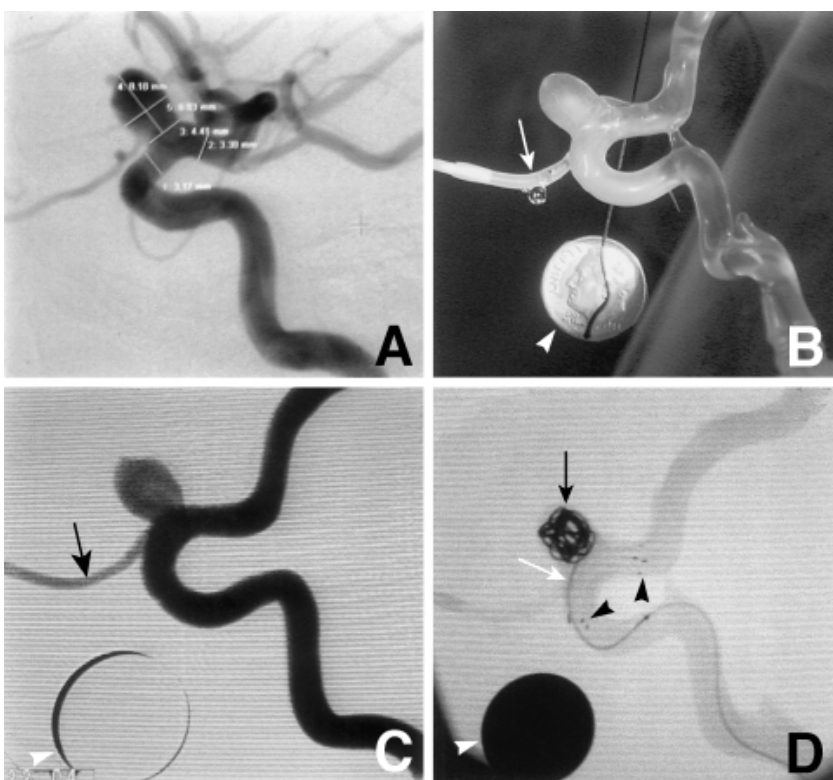

Fig. 3 A-C: Angiogram of an internal carotidophthalmic artery aneurysm (A), and photograph (B) and angiogram (C) of the silicone model showing the vascular course and shape of the aneurysm and neck were reproduced closely, including the ophthalmic artery (arrow). D: Angiogram showing introduction of the microcatheter (white arrow: Excelsior SL10; Boston Scientific Co., Ltd., Natick, Mass., U.S.A.) and stent (black arrowheads: Neuroform2; Boston Scientific Co., Ltd.), and coils (black arrow: Guglielmi detachable coil; Boston Scientific Co., Ltd.) into the model met stronger resistance compared with actual living vessels. white arrowhead: United States 5 cent coin, $21.2 \mathrm{~mm}$ diameter.

reduced a little by applying silicone lubricant.

The stroke model required linear reinforcement of the wax wire to prevent snapping in models of severe ICA or MCA stenosis (Fig. 4A). PTA and stenting procedure achieved much less dilation of the stenosis than expected (Fig. 4B), so the simulation could not provide the feeling of realistic manipulation.

The embolic occlusion model showed that all three embolic materials were appropriate for various simulations, such as PTA or penetration, but clay was harder to drain and so most easy to use. However, the simulations were very dissimilar from practice because of the friction between silicone and device.

\section{Silicone suture model}

The seams tended to leak, but not so heavily as to

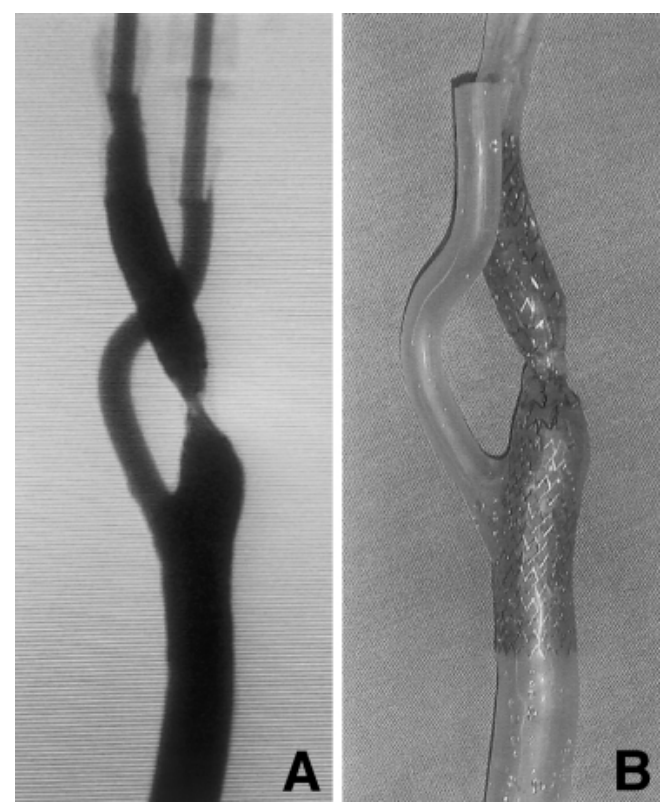

Fig. 4 Angiogram of the silicone model of cervical internal carotid artery stenosis demonstrating the realistic stenosis (A), and photograph showing inadequate dilation after stenting (B).

affect inspection and evaluation. This model might be the good choice for avoidance of tearing in the case of giant or small orifice aneurysms. However, the projection of the dome was not accurate enough, and the bumps caused by the sutures tended to disturb the introduction of the tip of the microcatheter into the aneurysm dome.

\section{Animal vascular model}

The IC-OphA aneurysm model was made by sideto-end anastomosis between the ICA and external jugular vein without reproduction of branch vessels. The BA top and AcomA aneurysm models were made by side-to-end anastomosis between the two ICAs, and the external jugular vein to represent the aneurysm added to the anastomotic region (Fig. 5A). All models showed leaks from various seams, but none was heavy enough to affect inspection and simulation of treatment.

Circular, elliptical, and cylindrical aneurysms could be modeled. However, the $3 \mathrm{~mm}$ diameter of the parent artery was difficult to compare to the ICA or MCA (Fig. 5B). The animal vascular model was much less accurate than the conventional silicone model in respect of the aneurysm shape and diameter of vessels. On the other hand, the silicone model sheath provided an accurate representation of the vascular course. The feel of device use was 


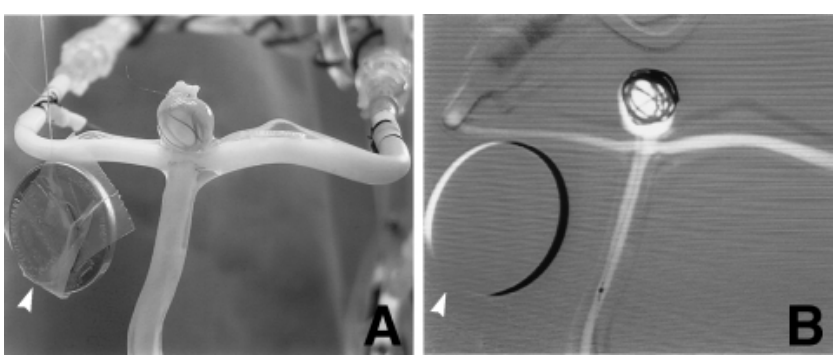

Fig. 5 A: Photograph of the animal vascular model of a top of the basilar artery aneurysm showing the elliptical dome shape, and the parent artery diameter of about $3 \mathrm{~mm}$. B: Angiogram of the model showing the unrealistic appearance, but the feel of device use was extremely close to the practical procedure, and no resistance or impact occurred during introduction of the microcatheter and coils. white arrowhead: United States 5 cent coin, $21.2 \mathrm{~mm}$ diameter.

extremely close to practical clinical manipulation, and devices could pass even sharp bends smoothly. Moreover, no friction and impact, unlike the conventional silicone model, occurred in the coiling procedure, and the sutures did not cause any resistance.

\section{Discussion}

The present models were made with reference to the images of clinical cases, so provided inferior reproducibility compared with the cadaver copy model, but could represent individual cases. We believe that individual models are very important for use in training instead of a standard model. The individual model is also effective as a preclinical simulation before the treatment of clinical cases. Moreover, the process to make the original wax model requires accurate evaluation of the shape and aspect of the aneurysm and the relationship with the surrounding vasculature, which provides important training in the assessment of the spatial relationship of the vasculature from angiography. Trainees using the standard model tend to look only at the technical solutions, rather than concentrating on other aspects, such as the anatomy, that are also relevant to the treatment. Therefore, the present model has additional training value.

The present modeling method attempts to reproduce all the fine detail of the original anatomy, to permit realistic simulation of endovascular procedures. The aneurysm model provided good representation of the shape and even the surround- ing fine branch vessels of about $1 \mathrm{~mm}$ diameter, except for the projection of the giant aneurysm dome. The present technique can model almost all types of aneurysm indicated for interventional treatment except for restricted types such as carotid cavernous fistula, etc. The stroke model allowed simulation of the introduction of the microcatheter, but severe stenosis was not easy to reproduce. The medium stenosis model allowed simulation of PTA and stenting procedures, but dilation was insufficient with the stent.

Previous training systems based on the silicone model have emphasized the advantages of the direct view, not fluoroscopy imaging, ${ }^{2,12)}$ which is hard to regard as practical and realistic training. Our system simulated the manual skill of the procedure with road mapping using angiography. Observation under fluoroscopy imaging and direct view was beneficial to grasp the spatial relationship between the lesions and devices.

The frictional resistance between silicone and device is the major disadvantage of the silicone model. The resistance is far firmer than that of the vasculature. Devices frequently could not pass curves in the model vessels, and the impact was also strong during aneurysm filling with coils. Therefore, the silicone model may be useful for training in the directions of devices and the procedure, but is far from adequate to experience the realistic clinical feel of devices and to assess capability. The hydrophilic polymer model reported by Sugiu et al. is adequate for basic training, ${ }^{12)}$ because catheters and devices can be introduced and placed smoothly with insignificant friction. However, the production process is too complicated to create the various models of individual case practically.

The animal vascular model could compensate for these disadvantages of the silicone model, as well as prevent unnecessary and expensive animal use. A more realistic training system could be established based on the animal vascular model. However, the absence of biological conditions would exclude biocompatibility testing. Several other important aspects of endovascular treatment also could not be simulated including the hydrodynamics of non-newtonian fluids, and the relationships between aneurysm morphology, hemodynamic stress, coil surface material, and activity of the clotting system. ${ }^{6,11)}$ Combinations of in vitro and in vivo models are essential for comprehensive research and training.

\section{References}

1) Gailloud P, Muster M, Piotin M, Mottu F, Murphy LJ, 
Fasel JH, RufenachiDA: In vitro models of intracranial arteriovenous fistulas for evaluation of new endovascular treatments. AJNR Am J Neuroradiol 20: 291-295, 1999

2) Gruber A, Bavinszki G, Killer M, Shameri A, Richling $B$ : In vitro training model for endovascular embolization of cerebral aneurysms. Minim Invasive Neurosurg 40: 121-123, 1998

3) Guglielmi G, Vinuela F, Dion J, Duckwiler G: Electrothrombosis of saccular aneurysms via endovascular approach. Part 2: Preliminary clinical experience. J Neurosurg 75: 8-14, 1991

4) Guglielmi G, Vinuela F, Sepetka I, Macellari V: Electrothrombosis of saccular aneurysms via endovascular approach. Part 1. Electrochemical basis, technique and experimental results. J Neurosurg 75: 1-7, 1991

5) Kerber CW, Heilman CB: Flow dynamics in the human carotid artery: I. Preliminary observations using a transparent elastic model. AJNR Am J Neuroradiol 13: 173-180, 1992

6) Kwan ES, Heilman CB, Roth PA: Endovascular packing of carotid bifurcation aneurysm with polyester fiber-coated platinum coils in a rabbit model. AJNR Am J Neuroradiol 14: 323-333, 1993

7) Malisch TW, Guglielmi G, Vinuela F, Duckwiler G, Gobin YP, Martin NA, Frazee JG: Intracranial aneurysms treated with the Guglielmi detachable coil: midterm clinical results in a consecutive series of 100 patients. J Neurosurg 87: 176-183, 1997

8) McDougall CG, Halbach VV, Dowd CF, Higashida RT, Larsen DW, Hieshima GB: Causes and management of aneurysmal hemorrhage occurring during embolization with Guglielmi detachable coils. J Neurosurg 89: 87-92, 1998

9) Piotin M, Mandai S, Murphy KJ, Sugiu K, Gailloud P, Martin JB, Rufenacht DA: Dense packing of cerebral aneurysms: an in vitro study with detachable platinum coils. AJNR Am J Neuroradiol 21: 757-760, 2000

10) Raymond J, Roy D: Safety and efficacy of endovascular treatment of acutely ruptured aneurysms. Neurosurgery 41: 1235-1246, 1997

11) Spetzger U, Reul J, Weis J, Bertalanffy H, Thron A, Gilsbach JM: Microsurgically produced bifurcation aneurysms in a rabbit model for endovascular coil embolization. J Neurosurg 85: 488-495, 1996

12) Sugiu K, Martin JB, Jean B, Gailloud P, Mandai S, Rufenacht DA: Artificial cerebral aneurysm model for medical testing, training, and research. Neurol Med Chir (Tokyo) 43: 69-73, 2003

Address reprint requests to: Y. Suzuki, M.D., Department of Neurosurgery, Ikegami General Hospital, 6-1-19 Ikegami, Oota-ku, Tokyo 146-8531, Japan. e-mail: yasu-suzuki-sn@ikegamihosp.jp

\section{Commentary}

This is an excellent paper dealing with the best way of training endovascular neurosurgeons to treat aneurysms. While classical training for surgeons has been, and still is, anatomical dissection, to confirm anatomy and become familiar with approaches, endovascular surgeons used to train on animal models ("iatrogenic" aneurysms in pigs) to learn how to deliver coils.

As the authors correctly point out, the animal model is excellent to train with the technical problems of the approach; however, it has a limited value for teaching how to face anatomical reconstruction of the vessel, because the anatomical variation is far from being comparable with clinical cases (i.e., the shape of the aneurysm is almost standard and the dimensions of the vessels are very much different from real cases). The silicone models, proposed in this paper, can reproduce more faithfully the real anatomy: in fact, they can be made using a clinical case as a template. Therefore, the trainees also have to confront problems related to anatomical variations, not just the technical difficulties of the approach. However, in silicone models, the approach is completely different as compared to human cases, because the catheter does not slide inside the tube as it does in a vessel coated with endothelium. The combination of animal models for the approach and of silicone models for treating the aneurysm gives the best training results.

I appreciated this work very much, and I think that Dr. Suzuki and colleagues provided a substantial contribution to the advance of clinical training.

Alessandro DuCATI, M.D. Ordinario di Neurochirurgia

Universita' di Torino Torino, Italy

This is a fascinating article by Suzuki and colleagues working at the University of Iowa, an outstanding institution where I was formerly a faculty member in Neurosurgery. They have described three laboratory methods for simulation of endovascular aneurysm treatment and have compared and contrasted the advantages and drawbacks of each. It should be essential reading for anyone interested in the current design of such systems.

This method differs from others in that the models were generated from 3-D angiographic images rather than cadaver specimens, and so they replicate true clinical conditions and have the variability seen in clinical practice. It is a great advantage for their scientific design to apply such principles.

Their discussion summarizes the work beautifully. Silicone models were anatomically accurate but gave 
less-than-ideal clinical "feel" during endovascular procedures. Animal-based material appears to give a better clinical simulation but proves to be more difficult in replicating the angiographic anatomy.

As the authors correctly state, fluoroscopic roadmapping simulation with a combination of silicone and animal-based models, with anatomy defined by clinical cases, is an exciting avenue of approach for the training of new endovascular neurosurgeons.

I congratulate these authors for an excellent study and a well-prepared manuscript.

Christopher M. LofTUS, M.D., F.A.C.S. Department of Neurosurgery Temple University School of Medicine Philadelphia, Pennsylvania, U.S.A. 